\title{
On the limitations of hybrid particle-in-cell for ion thruster plume simulations
}

Cite as: Phys. Plasmas 26, 103502 (2019); https://doi.org/10.1063/1.5111791

Submitted: 30 May 2019. Accepted: 04 September 2019. Published Online: 02 October 2019

Joseph Wang (D), and Yuan Hu (D)

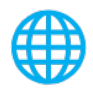

View Online

\section{ARTICLES YOU MAY BE INTERESTED IN}

Tutorial: Physics and modeling of Hall thrusters

Journal of Applied Physics 121, 011101 (2017); https://doi.org/10.1063/1.4972269

Vlasov code simulation of contact discontinuities

Physics of Plasmas 26, 102107 (2019); https://doi.org/10.1063/1.5100314

Electron kinetics in low-temperature plasmas

Physics of Plasmas 26, 060601 (2019); https://doi.org/10.1063/1.5093199

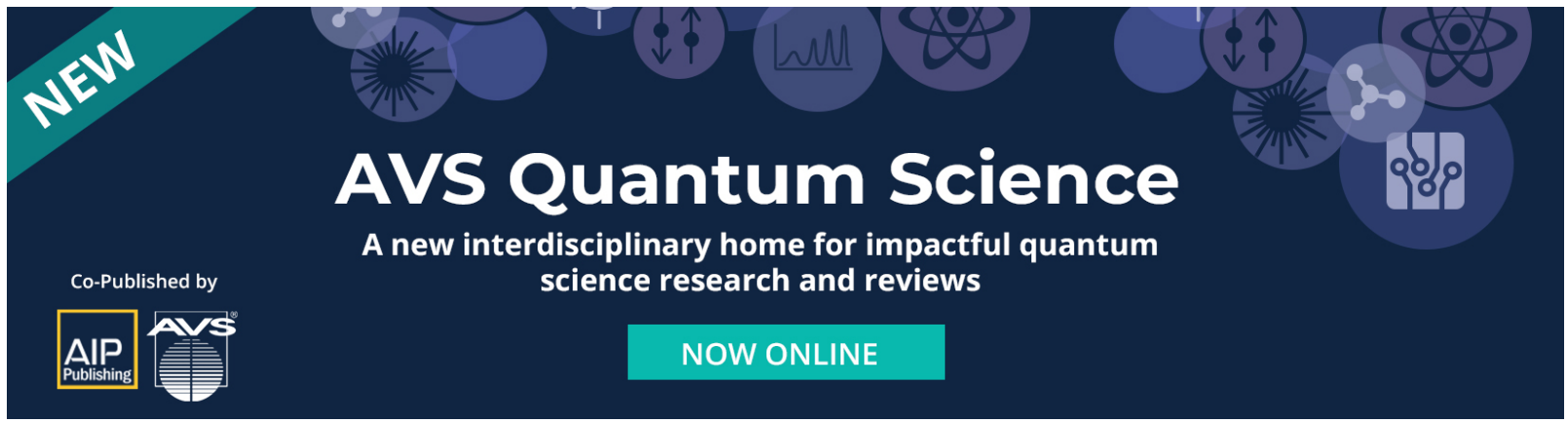




\title{
On the limitations of hybrid particle-in-cell for ion thruster plume simulations
}

\author{
Cite as: Phys. Plasmas 26, 103502 (2019); doi: 10.1063/1.5111791 \\ Submitted: 30 May 2019 - Accepted: 4 September 2019 . \\ Published Online: 2 October 2019
}

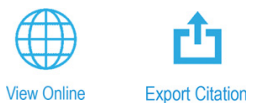

Joseph Wang ${ }^{1, a), b)}$ (D) and Yuan $\mathrm{Hu}^{1,2, b), c)}$ (iD

\author{
AFFILIATIONS \\ ${ }^{2}$ Institute of Mechanics, Chinese Academy of Sciences, Beijing 100190, China \\ a) Author to whom correspondence should be addressed: josephjw@usc.edu

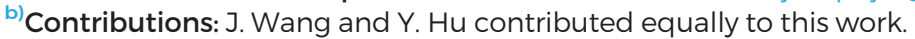 \\ ${ }^{c)}$ Electronic mail: yhu@imech.ac.cn
}

'Department of Astronautical Engineering, University of Southern California, Los Angeles, California 90089-1192, USA

\begin{abstract}
A commonly used approach to simulate ion thruster plumes is a hybrid particle-in-cell (PIC) with the Boltzmann relation to model electrons. The limitations of such an approach are investigated by benchmarking hybrid PIC against fully kinetic PIC simulations using the real $\mathrm{Xe}^{+}$to electron mass ratio $\left(m_{i} / m_{e}=241073\right)$. The results show that both full PIC and hybrid PIC lead to similar plume density structures but very different plume potentials. If one could ignore the ion beam neutralization region near the thruster exit, the main physics missing from a hybrid PIC plume model is that from the anisotropic electron characteristics. Including the beam neutralization region leads to further changes in electron temperature and plume potential. Compared to full PIC simulations, the hybrid PIC model results in an overestimation of the plume potential by about $30 \%-40 \%$ in the transverse direction for the simulations considered. Since the electrons in the plume are nonequilibrium and anisotropic, simply replacing the Boltzmann relation with the more general polytropic thermodynamic relation for electrons in a hybrid PIC plume model will not lead to a significant improvement in accuracy.
\end{abstract}

Published under license by AIP Publishing. https://doi.org/10.1063/1.5111791

\section{INTRODUCTION}

The plasma plume emitted by an electric propulsion (EP) thruster has been studied extensively (see Refs. 1-5 and references therein). In an ion thruster, cold beam ions are emitted from the thruster exit to provide the thrust, while thermal electrons are emitted from a neutralizer to neutralize the ion beam. The plume is mesothermal as the velocities of the beam ions and neutralizing electrons are such that $v_{t i} \ll v_{0} \ll v_{t e}$, where $v_{t i}, v_{0}$, and $v_{t e}$ denote the ion thermal velocity, plume velocity, and electron thermal velocity at the thruster exit, respectively. For typical EP application problems, the plume can also be considered collisionless because the collision mean free path is much larger than the plume characteristic length.

Almost all ion thruster plume simulation models are based on the hybrid particle-in-cell (PIC) method. In a hybrid PIC model, the ions are modeled as macroparticles, while the electrons are modeled as an equilibrium massless fluid to save computational time. A common approach is to treat the electrons as a massless, isothermal, ideal gas. Thus, from the electron state equation, $p_{e}=n_{e} k_{b} T_{e}$, and momentum equation,

$$
0 \simeq m_{e}\left(\frac{\partial \mathbf{v}_{e}}{\partial t}+\mathbf{v}_{e} \cdot \nabla \mathbf{v}_{e}\right)=e \nabla \Phi-\frac{1}{n_{e}} \nabla p_{e}
$$

where $m_{e}, n_{e}, \mathbf{v}_{e}, p_{e}$, and $T_{e}$ are the mass, density, velocity, pressure, and temperature for electrons, respectively, $k_{b}$ the Boltzmann constant, and $e$ the elementary charge, one obtains the Boltzmann relation between the electron density $n_{e}$ and the electric potential $\Phi$,

$$
n_{e}=n_{0} \exp \left[\frac{e\left(\Phi-\Phi_{0}\right)}{k_{b} T_{e 0}}\right]
$$

where $n_{0}, \Phi_{0}$, and $T_{e 0}$ are the plume density, potential, and electron temperature at the thruster exit, respectively. Equation (2) has been used in almost all plasma plume simulation models so far.

While the approach of hybrid PIC with the Boltzmann relation for electrons has been widely adopted, its limitations have not been carefully examined. An obvious issue is the application of the Boltzmann relation. The isothermal assumption in Eq. (2) allows an unlimited potential drop as the electron density decreases from the plume center to the ambient, a clearly unphysical result. Several studies have relaxed the isothermal assumption to include electron cooling in plume expansion by either assuming $T_{e}$ as a 
function of the position in Eq. $(2)^{6-11}$ or replacing Eq. (2) with the more general polytropic law,

$$
\frac{T_{e}}{n_{e}^{\gamma_{e}-1}}=\frac{T_{e 0}}{n_{e 0}^{\gamma_{e}-1}}
$$

where $\gamma_{e}$ is the polytropic coefficient for the electron gas. Note that $\gamma_{e}=1$ corresponds to the isothermal electron and results in the Boltzmann relation, Eq. (2). In a collision dominated gas, heat conduction is through interparticle collisions. From classical thermodynamics, the polytropic coefficient for the adiabatic expansion of an ideal monatomic gas is $\gamma_{e}=5 / 3$. However, in existing plume simulations utilizing either a position dependent $T_{e}$ or the polytropic law, the values of $\gamma_{e}$ were somewhat arbitrary. ${ }^{12}$ This is because the interparticle collisions are largely absent for the electrons in EP plumes, and thus the exact cooling mechanism for electrons has not been identified.

Another issue is the assumption that the electrons may be considered as an equilibrium fluid in a plasma plume. Based on the kinetic theory, the primary mechanism for electrons to reach a thermal equilibrium state is through particle-particle collisions, which are generally absent for the length scale of typical EP plume problems. Recently, full particle PIC simulations were applied to simulate plasma plume emissions. ${ }^{15-22} \mathrm{Hu}$ and $\mathrm{Wang}^{20-22}$ showed that the electrons in a plasma plume are nonequilibrium and the electron temperature is anisotropic. Hence, neither the Boltzmann relation equation (2) nor the more general polytropic law equation (3) appears to be a good assumption for modeling electrons in plasma plumes. ${ }^{21}$ However, the difference between hybrid PIC and full PIC has not been quantified under identical simulation settings.

Finally, the hybrid PIC approach cannot resolve ion beam neutralization and thus only simulates a quasineutral plume. Hence, a hybrid PIC plume model requires the plume potential $\Phi_{0}$ and electron temperature $T_{e 0}$ at the thruster exit in Eq. (2) to be provided by other means. Recent full particle PIC simulations of beam neutralization ${ }^{18,20,21}$ showed that $\Phi_{0}$ and $T_{e 0}$ are sensitively influenced by the beam neutralization region at the thruster exit. However, the effects of ignoring the beam neutralization region on a hybrid PIC plume model have not been evaluated.

Given the computational advantage of hybrid PIC over full particle PIC and the extensive amount of hybrid PIC plume simulation studies, it is important to understand the limitations of the Boltzmann relation based hybrid PIC model in EP applications and quantify the errors due to the assumption of the electron model. This paper presents such a study. We apply both the full PIC and the hybrid PIC to simulate the emission of a collisionless, mesothermal plasma plume. The hybrid PIC model is benchmarked against two full PIC models. The first full PIC model utilizes the identical simulation setup as the hybrid PIC to allow a direct comparison between the two methods under the same assumptions. The second one further takes beam neutralization into account. Both the Boltzmann relation equation (2) and the polytropic law equation (3) are evaluated using the full PIC results. Since xenon $(\mathrm{Xe})$ is the typical propellent used by ion thrusters and the ion mass can affect the electron cooling, ${ }^{22}$ the full PIC simulations are carried out using the actual $\mathrm{Xe}^{+}$ion to electron mass ratio, $m_{i} / m_{e}$ $=241073$. The rest of this paper is organized as follows: Sec. II describes the approach and simulation models. Section III compares full PIC and hybrid PIC simulation results. A summary and conclusions are presented in Sec. IV.

\section{SIMULATION MODELS}

The simulation setups are shown in Fig. 1. We consider a plasma plume consisting of cold beam ions and thermal electrons injected into the simulation domain along the $x$ direction. To simplify the analysis, the beam has a uniform density distribution at the emission surface. The simulation also does not include the small amount of low energy charge-exchange ions generated in an ion thruster plume due to computational constraints. To speed up the computation, the simulations are carried out in two-dimensions.

The plasma emitted by an ion thruster has an equal ion and electron current to prevent spacecraft charging. The plume is typically non-neutral near the thruster exit where beam neutralization takes place and becomes quasineutral a short distance further downstream. The details of the beam neutralization region depend on the neutralization design. Here, we consider two different plume emission setups, as illustrated in Fig. 1. In emission model 1, we ignore the beam neutralization region and assume that a well-neutralized plasma plume has already been established at the emission surface. Therefore, the plasma plume is both quasineutral, $n_{i 0}=n_{e 0}$, and current free, $J_{i 0}+J_{e 0}=0$, at the emission surface. In emission model 2, we impose only the current free condition at the emission surface. Because of the different velocity distributions for the cold beam ions and thermal electrons, the plume is initially non-neutral, $n_{i 0} \neq n_{e 0}$, near the emission surface. Beam neutralization is resolved during the simulation. We note that emission model 2 models the neutralization design using a thermionic emitter placed inside the ion beam for neutralization. ${ }^{23}$ The hybrid PIC only simulates a quasineutral plume, while the full PIC approach allows the inclusion of beam neutralization in the
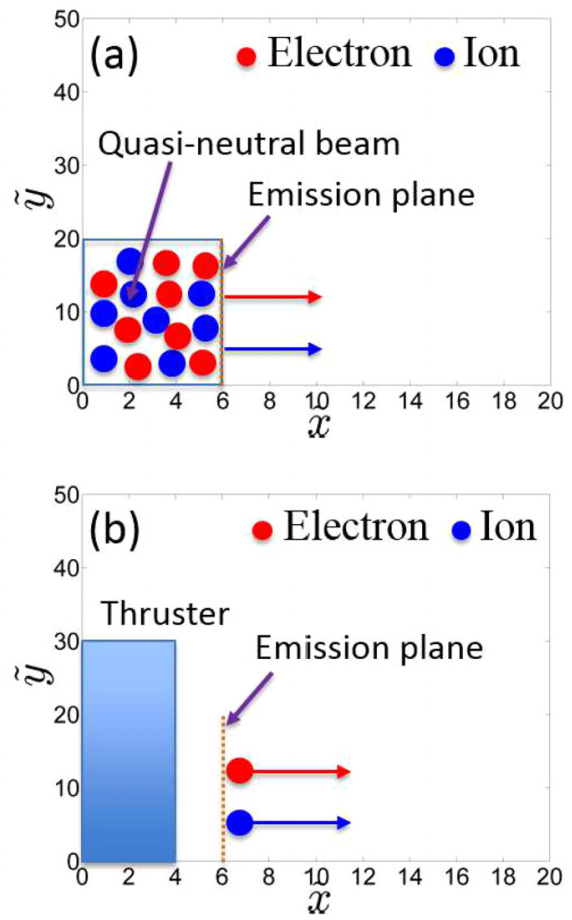

FIG. 1. Simulation setup. (a) Emission model 1: quasineutral beam emission. (b) Emission model 2: ion beam with thermal electrons emitted in the beam. 
plume. Hence, emission model 1 is used by both the hybrid PIC and full PIC simulations and emission model 2 is used only by the full PIC simulation.

In the full particle PIC model, both the electrons and the ions are treated as macroparticles. The dynamics of electrons and ions, the space charge, and the electric field are solved self-consistently from

$$
\begin{gathered}
\tilde{\nabla}^{2} \tilde{\Phi}=\left(\tilde{n}_{e}-\tilde{n}_{i}\right), \\
\frac{d}{d \tilde{t}}(\tilde{m} \tilde{\mathbf{v}})=\tilde{\mathbf{F}}, \quad \tilde{\mathbf{v}}=d \frac{\tilde{\mathbf{x}}}{d \tilde{t}} .
\end{gathered}
$$

In the hybrid PIC, only the ions are treated as macroparticles and the electrons are assumed to follow the Boltzmann relation, Eq. (2). Hence, the electron density $\tilde{n}_{e}$ in Eq. (4) is replaced by Eq. (2).

As the full PIC resolves the physics on the electron scale while the hybrid PIC only simulates the process on the ion scale, the full PIC uses dimensionless variables normalized by the electron plasma parameters, and the hybrid PIC uses those normalized by the ion plasma parameters. The normalization used for the full PIC is given by

$$
\begin{gathered}
\tilde{\mathbf{x}}=\frac{\mathbf{x}}{\lambda_{D 0}}, \quad \tilde{\mathbf{v}}=\frac{\mathbf{v}}{v_{t e 0}}, \quad \tilde{t}=t \omega_{p e 0}, \\
\tilde{m}_{i, e}=\frac{m_{i, e}}{m_{e}}, \quad \tilde{n}_{i, e}=\frac{n_{i, e}}{n_{0}}, \quad \tilde{\Phi}=\frac{e \Phi}{k_{b} T_{e 0}},
\end{gathered}
$$

and the normalization for the hybrid PIC is given by

$$
\begin{gathered}
\tilde{\mathbf{x}}=\frac{\mathbf{x}}{\lambda_{D 0}}, \quad \tilde{\mathbf{v}}=\frac{\mathbf{v}}{C_{s 0}}, \quad \tilde{t}=t \omega_{p i 0}, \\
\tilde{m}_{i}=\frac{m_{i}}{m_{i}}=1, \quad \tilde{n}_{i, e}=\frac{n_{i, e}}{n_{0}}, \quad \tilde{\Phi}=\frac{e \Phi}{k_{b} T_{e 0}} .
\end{gathered}
$$

In Eqs. (6) and (7), the subscript " 0 " denotes the condition at the emission exit, $\lambda_{D 0}=\sqrt{\epsilon_{0} k_{b} T_{e 0} / n_{0} e^{2}}, \omega_{p e 0}=\sqrt{n_{0} e^{2} / \epsilon_{0} m_{e}}$, and $\omega_{p i 0}$ $=\sqrt{n_{0} e^{2} / \epsilon_{0} m_{i}}$ are the Debye length, electron plasma frequency, and ion plasma frequency, respectively, $v_{t e 0}=\sqrt{k_{b} T_{e 0} / m_{e}}$ is the electron thermal velocity, and $C_{s 0}=\sqrt{k_{b} T_{e 0} / m_{i}}$ is the ion acoustic velocity.

In an ion thruster, the propellant ion temperature is close to the thruster wall temperature at a few hundred Kelvin (a few percent of $1 \mathrm{eV})$, and the electron temperature measured near the thruster exit is a few electron volts. ${ }^{2,3}$ At an acceleration voltage of about $1 \mathrm{kV}$, the Mach number for Xenon beam ions is about 20-30. Hence, for all the simulations in this paper, we take the ion temperature to electron temperature ratio at the exit to be $T_{i 0} / T_{e 0}=0.01$ and the Mach number of the beam ions to be $M_{0}=20$. (This corresponds to a Xenon ion thruster plume with $v_{o} \simeq 35 \mathrm{~km} / \mathrm{s}, T_{i 0} \simeq 0.045 \mathrm{eV}$, and $T_{e 0} \simeq 4.5 \mathrm{eV}$.) Accordingly, the beam velocity is set to be $v_{0} / v_{t e 0}=0.040734$. The size of the initial beam in $y$ is taken to be $R_{\text {beam }}=20 \lambda_{D 0}$.

In the hybrid PIC simulation, the injection of beam ions from the thruster exit is straightforward. In full particle PIC simulations, the injection of ions and electrons is handled as follows. In emission model 1 , we consider that a plasma reservoir is located in the region of $(\tilde{x}, \tilde{y})=(0-6,0-20)$. Both the electrons and ions are loaded as drifting Maxwellian distributions with the same drifting velocity in the reservoir and are injected from the emission surface at $\tilde{x}_{\text {emission }}$ $=6, \tilde{y}_{\text {emission }}=0-20$ according to the state of the plasma reservoir at each simulation step. The thermal electrons in the reservoir adjust themselves to generate a quasineutral, current-free plume at the emission surface. ${ }^{21}$ The electric potential in the region of the reservoir is fixed at zero. In emission model 2, a thruster body is set to occupy the region of $(\tilde{x}, \tilde{y})=(0-4,0-30)$, and the plasma emission surface is at the same location as that in emission model $1\left(\tilde{x}_{\text {emission }}=6, \tilde{y}_{\text {emission }}\right.$ $=0-20)$. The potential of the thruster body is fixed at zero. The ions are injected as a drifting Maxwellian distribution from the emission surface. The electrons are loaded as a stationary Maxwellian distribution at the emission surface and are injected following the free molecular effusion process. ${ }^{21}$ Because the electron thermal velocity is much larger than the ion drifting velocity, the current-free condition leads to a non-neutral plasma near the emission surface.

Simulations need to run for a time duration long enough to achieve a steady state plume near the thruster. The full PIC simulations also need to be terminated far before the beam front reaches the boundary of simulation domain. This is because the electron distribution in the expansion is not known a priori, and thus it is difficult to accurately predetermine the particle boundary condition in the full PIC simulation. These two constraints determine the domain size and simulation duration. The simulation domain used is $\tilde{L}_{x} \times \tilde{L}_{y}=2000$ $\times 1000$, with a mesh size of $\Delta \tilde{x}=\Delta \tilde{y}=1$. The symmetric boundary condition is applied at the plane of $\tilde{y}=0$ for the field as well as the particles. The zero Neumann boundary condition for the electric field is applied at other boundaries of the simulation domain. The simulations are run for a duration up to $t \omega_{p i 0}=45$. The time step used for the fully kinetic PIC simulation is $\Delta \tilde{t}=\Delta t \omega_{p e 0}=0.05$ and that for the hybrid PIC simulation is $\Delta \tilde{t}=\Delta t \omega_{p i 0}=0.01$. Note that $\omega_{\text {pe0 }} / \omega_{p i 0}=\sqrt{m_{i} / m_{e}} \simeq 491$, and so the total simulation steps are 441900 in the fully kinetic PIC simulation and 4500 in the hybrid PIC simulation. The transient plume evolution was examined throughout the simulation. We find that the plume within the region of $\tilde{x} \leq 750$ reached the steady state at $t \omega_{p i 0} \simeq 40$, well before the simulation was terminated.

For the simulations considered, the number of PIC time steps in a hybrid PIC simulation is $4500 / 441900 \simeq 10^{-2}$ of that in a full PIC simulation. As the hybrid PIC only simulates the ion species and uses half as many macroparticles as those in a full PIC simulation, its particle push time is about half of that in full PIC. On the other hand, the hybrid PIC solves the nonlinear Poisson's equation using Newton's iteration method, which is typically about 7-8 times slower than the linear Poisson's solver used in the full PIC for our cases. As a result, one step in hybrid PIC is about 3-4 times more time-consuming than one step in full PIC. Hence, in this study, the overall speedup of hybrid PIC over full PIC is about a factor of 25-30.

\section{RESULTS AND DISCUSSION}

This section compares results from three different sets of simulations. Hereafter, we refer the hybrid PIC and the full particle PIC simulations using emission model 1, shown in Fig. 1(a), as simulation A and simulation B, respectively, and refer the full particle PIC simulation using emission model 2, shown in Fig. 1(b), as simulation C. All results shown in this section are at $t \omega_{p i 0}=40\left(t \omega_{p e 0} \simeq 19640\right)$.

Figure 2 shows the ion and electron number density contours obtained from the three different simulations. The density contours from all three simulations show a similar pattern. In simulation $\mathrm{C}$, the plume is initially non-neutral near the emission surface but becomes well-neutralized starting at about $3 \lambda_{D 0}$ downstream. The initial 

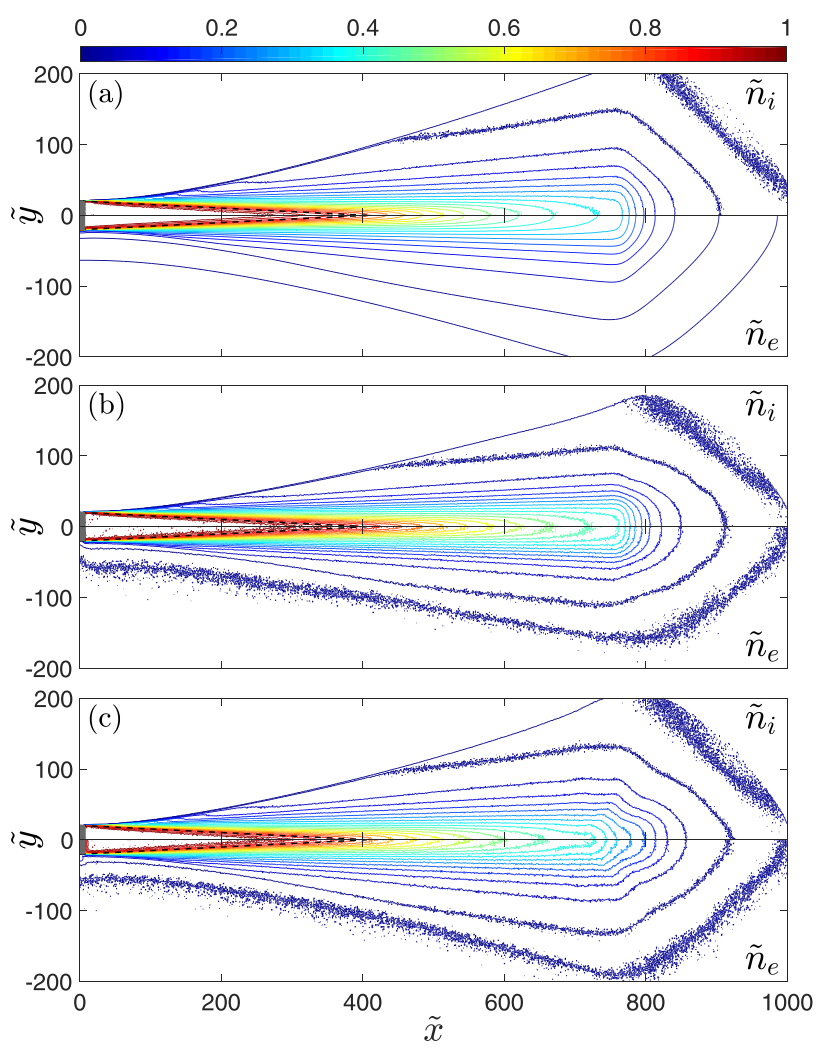

FIG. 2. Density contours. (a) Simulation A. (b) Simulation B. (c) Simulation C. The upper and lower panels in each subfigure show the ion and electron density, respectively. The black dashed line shows the analytical Mach line.

non-neutral region corresponds to a thin sheath at the exit surface. The plume downstream of the sheath is similar to that of Simulations $\mathrm{A}$ and $\mathrm{B}$. The neutralization process for emission model 2 was discussed in detail in Wang et al. ${ }^{18}$

The expansion of a quasineutral mesothermal plasma has been studied both analytically and through hybrid PIC simulations. ${ }^{24,25}$ In a quasineutral mesothermal plasma expansion, the boundary that separates the unperturbed plasma from the expansion region is the Mach line computed based on the Mach number of the undisturbed plasma. The Mach line is the first characteristic line of the expansion fan, and the slope of the Mach line is ${ }^{24,25}$

$$
k=\tan (\theta), \quad \theta \simeq \sin ^{-1}\left(\frac{1}{M_{0}}\right) .
$$

The Mach line originating from the edge of the beam emission surface, $\left(\tilde{x}_{0}, \tilde{y}_{0}\right)=(6,20)$, and based on the Mach number at the emission surface $M_{0}$ is also plotted as the black dashed line in Fig. 2 (as well as in Figs. 3, 6, and 7). The Mach line intersects the beam center axis at $\tilde{x}-\tilde{x}_{0} \simeq 400$ for $M_{0}=20$. In the simulations, the inner expansion boundary qualitatively agrees with this result. The region inside the Mach line is the unperturbed plume core region where the plasma condition is the same as that at the starting location of the quasineutral plume.


FIG. 3. Density error of hybrid PIC against full PIC. (a) Simulation A vs simulation B. (b) Simulation A vs simulation C. The upper and lower panels in each subfigure show the ion and electron densities, respectively. The black solid lines show representative density values from hybrid PIC. The black dashed line shows the analytical Mach line.

To quantitatively compare the plume density contours from the three simulations, Fig. 3 further shows the local density error of hybrid PIC against the full PIC results. The local density error of hybrid PIC is calculated as

$$
\begin{aligned}
& \tilde{n}_{i, e r r}=\frac{\left|\tilde{n}_{i, h y b r i d}-\tilde{n}_{i, \text { full }}\right|}{\tilde{n}_{i, \text { hybrid }}}, \\
& \tilde{n}_{e, \text { err }}=\frac{\left|\tilde{n}_{e, h y b r i d}-\tilde{n}_{e, \text { full }}\right|}{\tilde{n}_{e, \text { hybrid }}},
\end{aligned}
$$

where the subscripts hybrid and full represent the hybrid and full PIC results, respectively. The plasma number densities of representative values from the hybrid PIC simulation are also superimposed. The results are shown for $\tilde{n}_{i}>10^{-3}$ and $\tilde{n}_{e}>10^{-3}$, which correspond to at least 4 particles per cell. The error contours are shown only for $\tilde{n}_{i, e r r}>10 \%$ and $\tilde{n}_{e, e r r}>10 \%$, respectively. The differences between the hybrid PIC and both full PIC simulations are found to remain small in the inner plume region. The comparison between simulation A and simulation B (full PIC, emission model 1) in Fig. 3(a) shows 

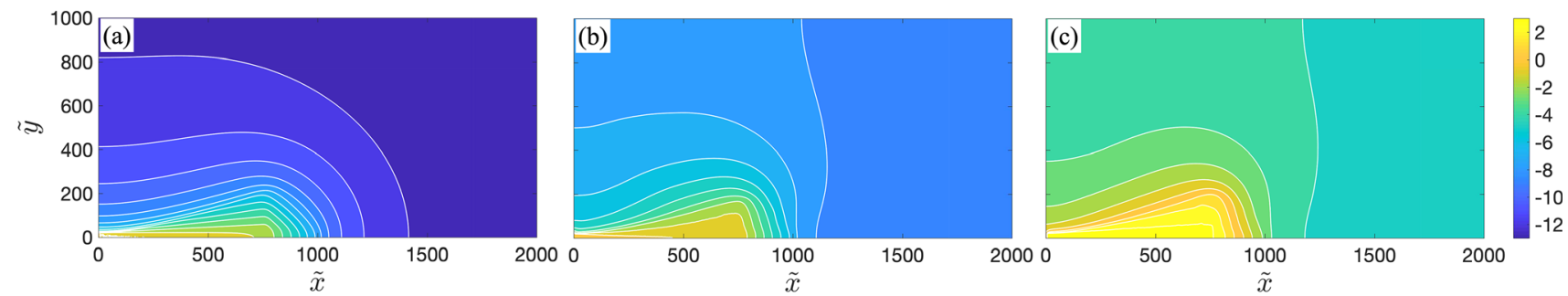

FIG. 4. Potential contours. (a) Simulation A. (b) Simulation B. (c) Simulation C.

that the relative error remains at $\tilde{n}_{i, e r r} \leq 10 \%$ and $\tilde{n}_{e, e r r} \leq 10 \%$ until $\tilde{n}_{i, h y b r i d} \sim 0.2$ and $\tilde{n}_{e, h y b r i d} \sim 0.2$, respectively. In the outer plume region where $\tilde{n}_{i, h y b r i d} \leq 0.1$ and $\tilde{n}_{e, \text { hybrid }} \leq 0.1$, the error of the hybrid PIC simulation becomes $\tilde{n}_{i, e r r} \geq 30 \%$ and $\tilde{n}_{e, e r r} \geq 30 \%$. It is interesting to find that simulation $\mathrm{A}$ shows better agreement with simulation C (full PIC, emission model 2) than simulation B. When compared with simulation C, the hybrid PIC error remains at $\tilde{n}_{i, \text { err }} \leq 10 \%$ and $\tilde{n}_{e, \text { err }} \leq 10 \%$ until approximately $\tilde{n}_{i, h y b r i d} \sim 0.05$ and $\tilde{n}_{e, \text { hybrid }} \sim 0.05$, respectively, as seen in Fig. 3(b).

Figure 4 compares the electric potentials obtained from the three simulations. Unlike the agreement shown in the density distribution, the potential distributions are quite different between hybrid PIC and full PIC. Since the potential of plume is small compared to the kinetic energy of the beam ions, the difference in potential distribution does not result in a significant difference in the plume density distribution. 1D potential profiles are further compared. Figure 5(a) shows the $1 \mathrm{D}$ profile along the center axis of the beam. The profiles from simulation A and simulation B are almost the same, showing an unperturbed core and plume expansion. As discussed by $\mathrm{Hu}$ and Wang, ${ }^{22}$ plume expansion may be further distinguished along the beam direction into a quasisteady expansion, a self-similar expansion, and an electron front region. The potential profile from simulation $\mathrm{C}$ differs from that of simulations $\mathrm{A}$ and $\mathrm{B}$ in that a sheath is established at the thruster exit. Since $n_{i 0}>n_{e 0}$ in the beam neutralization region at the thruster exit, simulation $\mathrm{C}$ also shows a higher potential in the beam center. The maximum potential in the plume core is about $\Phi_{\max } /\left(k_{b} T_{e 0} / e\right) \simeq 5$. It is worth noting that this value is in the same range of the measured plume potential for ion thrusters. ${ }^{3,23}$ In simulation $C$, the exit sheath slightly reduces the ion beam velocity and increases the electron temperature for the quasineutral plume. This results in a slightly smaller Mach number. However, the shape of the potential profile of simulation $C$ is similar to that of Simulations A and B downstream of the exit sheath. Hence, the beam neutralization region affects the plume potential magnitude but does not qualitatively affect the expansion along the beam direc-

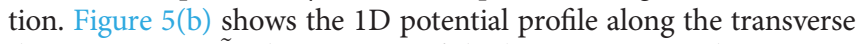
direction at one $\tilde{R}_{t}$ downstream of the beam exit. In order to compare the electric field in the plume expansion region, we plot the potential difference with respect to the plume centerline at the same downstream location, $\Delta \tilde{\Phi}=\tilde{\Phi}-\tilde{\Phi}_{c}=\tilde{\Phi}(\tilde{x}, \tilde{y})-\tilde{\Phi}(\tilde{x}, \tilde{y}=0)$, where $\tilde{x}$ is taken within the unperturbed core region. While the potential profiles from the full particle simulations (Simulations B and $\mathrm{C}$ ) have a similar slope, the hybrid PIC simulation (simulation A) leads to a significantly larger potential drop. This is because Eq. (2) allows an unbounded potential drop as the plume density decreases.
We use the potential drop from the unperturbed plume center to the ambient along the transverse direction to define the relative error in potential for hybrid PIC,

$$
\tilde{\Phi}_{\text {err }}(\tilde{y})=\frac{\Delta \tilde{\Phi}_{\text {hybrid }}-\Delta \tilde{\Phi}_{\text {full }}}{\Delta \tilde{\Phi}_{\text {hybrid }}} .
$$

For the simulation setups considered here, the full PIC profiles asymptote at $\tilde{y} \sim 800=40 \tilde{R}_{\text {beam }}$. We find that the hybrid PIC error in potential is about $\tilde{\Phi}_{\text {err }}\left(\tilde{y}=40 \tilde{R}_{\text {beam }}\right) \simeq 38 \%$ when compared to
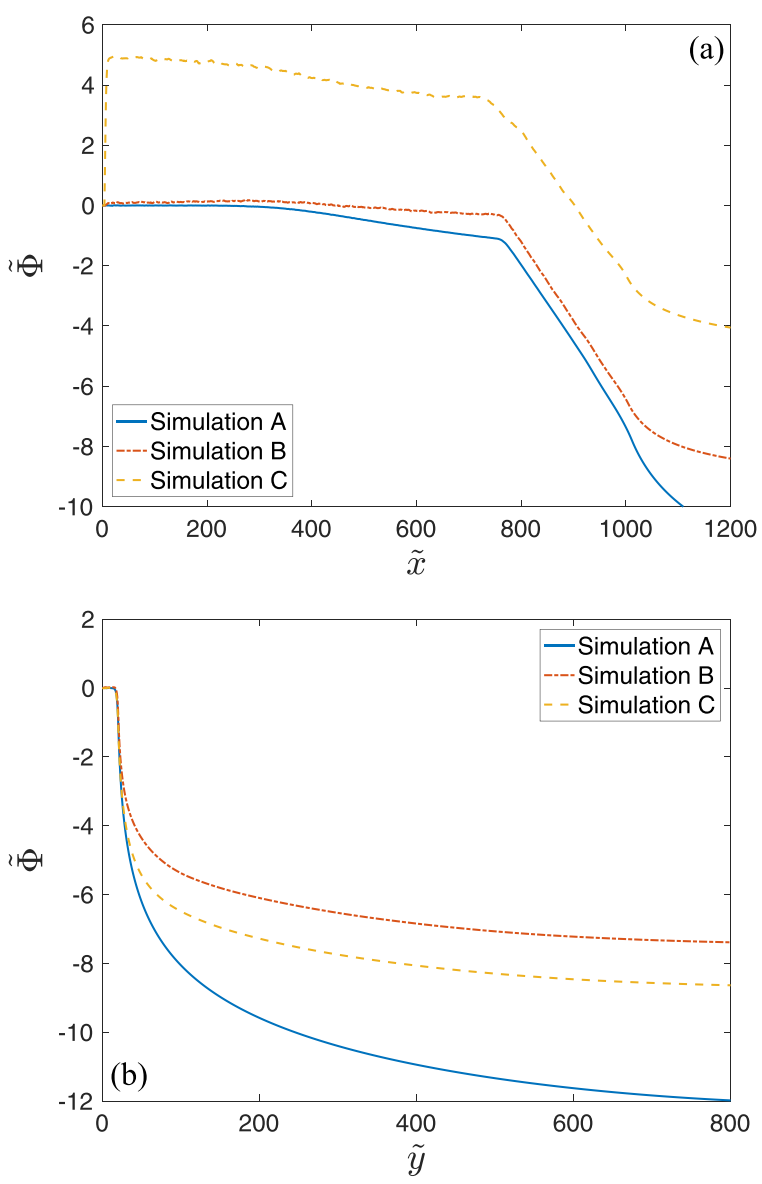

FIG. 5. 1D potential profiles. (a) Along the beam center axis ( $x$-axis) at $\tilde{y}=0$. (b) Along transverse direction ( $y$-axis) at $\tilde{x}-\tilde{x}_{\text {emission }}=20\left(=\tilde{R}_{\text {beam }}\right)$. 
simulation B and the hybrid error is about $\tilde{\Phi}_{\text {err }}\left(\tilde{y}=40 \tilde{R}_{\text {beam }}\right) \simeq 29 \%$ when compared to simulation C. These values may be used as a benchmark for the global error in the potential determined using hybrid PIC.

The comparisons show that the results from simulation A agree better with those from simulation $\mathrm{C}$ than the results from simulation B. We find that this may be attributed to the difference in electron temperature. Figure 6 shows the electron temperature contours obtained from simulation B and simulation $\mathrm{C}$. The electron temperature $\tilde{T}_{e}$ is derived from the second velocity moment of electrons averaged over the macroparticles within each cell. The normalized 2-D electron temperature at each mesh point is calculated from

$$
\begin{aligned}
\tilde{T}_{e, j}(\mathrm{ix}, \mathrm{iy}) & =\tilde{v}_{t e, j}^{2}(\mathrm{ix}, \mathrm{iy})=\left\langle\tilde{v}_{j}^{2}\right\rangle_{e}-\left\langle\tilde{v}_{j}\right\rangle_{e}^{2}, \\
\left\langle\tilde{v}_{j}^{n}\right\rangle_{e} & =\sum_{p=1}^{N_{e}} \tilde{v}_{p}^{n}(j) / N_{e},
\end{aligned}
$$

where $j=x, y, n=1,2$, and $N_{e}$ is the number of electron macroparticles in a given cell (ix, iy). The statistical noise of $\tilde{T}_{e, j}$ in each cell is proportional to $1 / \sqrt{N_{e}}$. To reduce the interference of the statistical noise, the calculations of Eq. (12) were carried out only for those cells with $N_{e} \geq 16$.

Figure 6 shows that the electron temperature obtained from full particle PIC simulations is not isothermal. Moreover, $\tilde{T}_{e, x}$ and $\tilde{T}_{e, y}$ behave very differently. We find that $\tilde{T}_{e, y}$ decreases as the plume expands outward, while $\tilde{T}_{e, x}$ varies much more slowly (though $\tilde{T}_{e, x}$ for simulation $\mathrm{C}$ is found to have a larger variation). Outside the plume core region, the potential drop is mostly along the $y$-direction. As the electrons expand outward in the $y$-direction, their kinetic energy is spent to overcome the electrostatic potential, leading to a decrease in $\tilde{T}_{e, y}$. In the $x$-direction, since there is neither electrostatic force nor
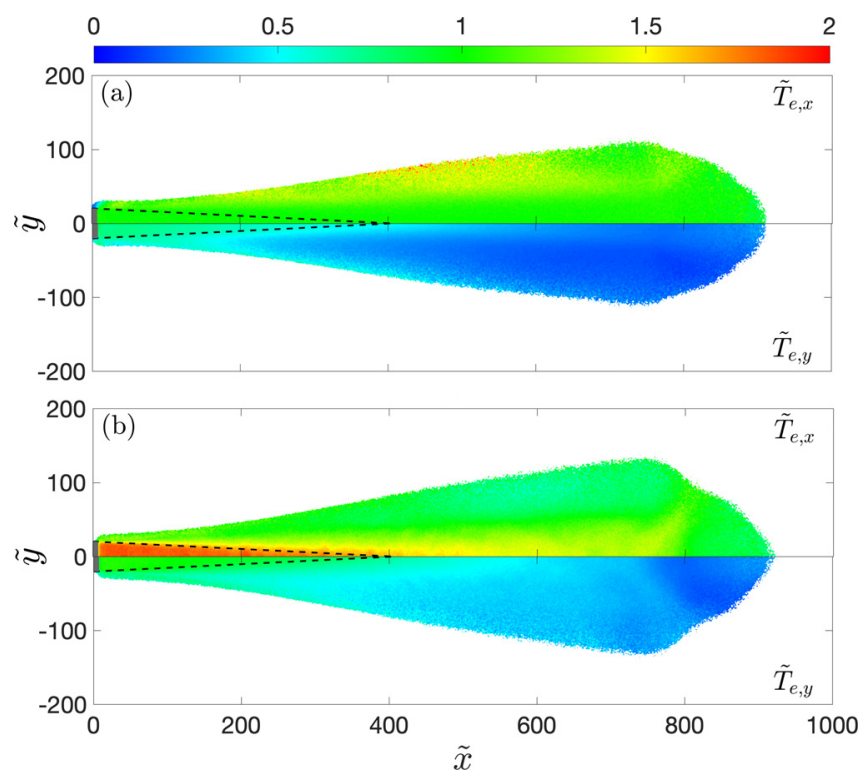

FIG. 6. Electron temperature contour from full PIC simulations. (a) Simulation B. (b) Simulation C. The upper and lower panels in each subfigure show $\tilde{T}_{e, x}$ and $\tilde{T}_{e, y}$, respectively. collisions to substantially affect electron thermal velocities, $\tilde{T}_{e, x}$ remains approximately constant. The electrons have the same initial temperature in both emission model 1 and emission model 2. However, because the plasma is non-neutral at the emission surface in emission model 2, a sheath is formed at the thruster exit and accelerates the emitted electrons to a higher energy. ${ }^{21}$ Hence, the main difference between simulation $\mathrm{B}$ and simulation $\mathrm{C}$ is that $\tilde{T}_{e, x}$ in simulation $\mathrm{C}$ is significantly higher inside the main beam. The higher $\tilde{T}_{e}$ indicates that there is more electron thermal energy to drive the expansion of the quasineutral plume in simulation C than that in simulation B. In the Boltzmann electron based hybrid PIC (simulation A), the isothermal assumption implies that the electron thermal energy available for plume expansion can be considered as infinite. This may explain why simulation A shows better agreement with simulation $\mathrm{C}$ than simulation $\mathrm{B}$.

One may try to use a polytropic relation to fit each component of the electron temperature. From the polytropic relation, Eq. (3), the polytropic coefficient can be written as

$$
\gamma_{e, j}=1+\frac{\log \left(\tilde{T}_{e, j} / \tilde{T}_{e 0}\right)}{\log \left(\tilde{n}_{e} / \tilde{n}_{e 0}\right)},
$$

where the subscript $j=x$ or $y$ represents the different components of the temperature and the corresponding polytropic coefficient. Figures 7 (a) and 7(b) show the polytropic coefficient contours in the plume expansion region obtained from simulation $\mathrm{B}$ and simulation $\mathrm{C}$, respectively. It is clear that the anisotropy in electron temperature leads to anisotropic polytropic coefficients. The polytropic coefficient fits are also influenced by the electron-ion mixing region near the emission surface. For simulation B, we find $\gamma_{e, x} \sim 1$ and $\gamma_{e, y} \sim 1.1-2.5$. For simulation $\mathrm{C}$, we find $\gamma_{e, x} \sim 1-1.5$ and $\gamma_{e, y} \sim 1.1-2.5$. Hu and Wang ${ }^{21}$ examined the local electron velocity distribution functions


FIG. 7. Polytropic coefficient $\gamma$ contour from full PIC simulations. (a) Simulation B. (b) Simulation C. The upper and lower panels in each subfigure show $\tilde{\gamma}_{e, x}$ and $\tilde{\gamma}_{e, y}$, respectively. 
(VDFs) obtained from the full particle PIC and showed that the electron VDFs in the plume deviate significantly from the Maxwellian distribution. This suggests that neither the Boltzmann relation nor the polytropic law may provide an accurate model for electrons. Hence, simply replacing the Boltzmann relation equation (2) with the polytropic relation equation (3) in a hybrid PIC simulation would not lead to a significant improvement in accuracy.

It is noted that some recent experimental observations of VASIMR and pulsed plasma thruster plumes attribute the electron cooling mechanism to that of ambipolar diffusion. ${ }^{26,27}$ The results presented here suggest that, while the cooling in the transverse direction exhibits features similar to ambipolar diffusion, the overall electron cooling in an ion thruster plume is more complex due to the strongly anisotropic characteristics.

\section{CONCLUSIONS}

Hybrid PIC is a commonly used approach in EP simulation studies. In this paper, both hybrid PIC and fully kinetic PIC simulations are carried out to quantify the limitations of hybrid PIC modeling of ion thruster plume emission. The hybrid PIC simulation adopted the commonly used assumption that the electrons may be modeled using the Boltzmann relation. The hybrid PIC model was benchmarked against two full PIC models, one utilizes an identical simulation setup of a quasineutral plume and the other further includes beam neutralization at the thruster exit. The full particle PIC simulations are carried out using the real $\mathrm{Xe}^{+}$to electron mass ratio $\left(m_{i} / m_{e}=241073\right)$. The results show that the hybrid PIC and full particle PIC simulations produce a qualitatively similar plume density distribution but very different electric fields. If one could ignore the effect of the beam neutralization region in the plume, the main physics that is missing from the hybrid PIC model is the anisotropic electron temperature due to electrons being nonequilibrium. In a quasineutral plume, the full PIC simulation shows that the electrons are near isothermal along the beam direction but exhibit significant cooling in the transverse direction. Since the electrons are nonequilibrium and anisotropic, neither the Boltzmann relation nor the polytropic law represents an accurate approximation for the electrons in a plasma plume. Under an identical simulation setting, the Boltzmann relation based hybrid PIC simulation leads to more than $30 \%$ local error in plume density in the outer plume region and overestimates the potential drop from the unperturbed plume core region to ambient in the transverse direction by about $40 \%$. Including the beam neutralization region leads to a sheath at the thruster exit, an initial increase in the electron temperature along the beam direction and electron cooling further downstream, and an increase in the plume potential in the unperturbed beam core region. When compared to the full PIC with beam neutralization modeled, the hybrid PIC has a local error of more than $10 \%$ in plume density in the outer plume region and a global error of about $30 \%$ in the potential drop from the plume center to ambient in the transverse direction. For the two full particle PIC simulation setups considered here, the result from the one including beam neutralization (beam emission model 2) agrees somewhat better with the Boltzmann relation based hybrid PIC. This is because the neutralization process provides more heating of the electrons at the thruster exit to drive plume expansion further downstream. Whether this is a general conclusion for other simulation setups will need to be investigated further.
The comparisons summarized above suggest that, in general, the accuracy of a hybrid PIC based prediction is acceptable in the inner plume region but unacceptable in the outer plume region where $\tilde{n}_{i}<0.1$. This finding is particularly relevant for hybrid PIC models of plume-spacecraft interactions. The simulations presented here only considered the emission of the primary beam and do not include the small amount of low energy charge-exchange (CEX) ions generated in the plume. However, as the hybrid PIC over-predicts the potential drop from plume to ambient by about $30 \%$ to $40 \%$, such an error in the electric field would also lead to an over-estimation of CEX ion backflow if the CEX ions were included. Hence, the application of the computationally more expansive full PIC may be unavoidable if one wishes to resolve accurately the interactions between CEX ions and spacecraft. The limitations of hybrid PIC in CEX ion backflow simulations will be quantified in a future study.

Hybrid PIC simulations require significantly less computational resources than fully kinetic PIC. We find that, in order to retain the hybrid PIC approach while minimizing the error in ion thruster plume simulations, one needs to adopt an electron model that takes into account the anisotropic electron characteristics in the plume and a simulation setup that takes into account the effects of beam neutralization at the thruster exit. Such an electron model will need to be investigated in a future study. The current study focused on the electron thermodynamics as the ions are much colder than the electrons in the simulation. Future studies will also need to consider higher $T_{i 0} / T_{e 0}$ ratios where the ion thermodynamics is also likely to play a role in the expansion process.

\section{ACKNOWLEDGMENTS}

The simulations in this paper were performed at the Center for High-Performance Computing of the University of Southern California.

\section{REFERENCES}

${ }^{1}$ R. I. S. Roy and D. E. Hastings, "Three-dimensional modeling of dual ion-thruster plumes for spacecraft contamination,” J. Spacecr. Rockets 33, 519-524 (1996).

${ }^{2}$ J. Wang, D. E. Brinza, D. T. Young, J. E. Nordholt, J. E. Polk, M. D. Henry, R. Goldstein, J. J. Hanley, D. J. Lawrence, and M. Shappirio, "Deep space one investigations of ion propulsion plasma environment," J. Spacecr. Rockets 37, 545-555 (2000).

${ }^{3}$ J. Wang, D. Brinza, and M. Young, “Three-dimensional particle simulations of ion propulsion plasma environment for Deep Space 1," J. Spacecr. Rockets 38, 433-440 (2001).

${ }^{4}$ I. D. Boyd, “Review of Hall thruster plume modeling," J. Spacecr. Rockets 38, 381-387 (2001).

${ }^{5}$ J. Wang, Y. Cao, R. Kafafy, J. Pierru, and V. K. Decyk, "Simulations of ion thruster plume-spacecraft interactions on parallel supercomputer," IEEE Trans. Plasma Sci. 34, 2148-2158 (2006).

${ }^{6}$ R. I. S. Roy, D. E. Hastings, and N. A. Gatsonis, "Ion-thruster plume modeling for backflow contamination," J. Spacecr. Rockets 33, 525-534 (1996).

${ }^{7}$ N. A. Gatsonis and X. Yin, "Hybrid (particle/fluid) modeling of pulsed plasma thruster plumes," J. Propul. Power 17, 945-958 (2001).

${ }^{8}$ D. B. VanGilder, I. D. Boyd, and M. Keidar, "Particle simulations of a Hall thruster plume," J. Spacecr. Rockets 37, 129-136 (2000).

${ }^{9}$ F. Taccogna, D. Pagano, F. Scortecci, and A. Garulli, "Three-dimensional plume simulation of multi-channel thruster configuration," Plasma Sources Sci. Technol. 23, 065034 (2014).

${ }^{10} \mathrm{I}$. D. Boyd and J. T. Yim, "Modeling of the near field plume of a Hall thruster," J. Appl. Phys. 95, 4575-4584 (2004).

${ }^{11}$ C. Cai, "Numerical studies on plasma plume flows from a cluster of electric propulsion devices," Aerosp. Sci. Technol. 41, 134-143 (2015). 
${ }^{12} \mathrm{M}$. Merino and E. Ahedo, "Influence of electron and ion thermodynamics on the magnetic nozzle plasma expansion,” IEEE Trans. Plasma Sci. 43, 244-251 (2015).

${ }^{13}$ M. Martinez-Sanchez, J. Navarro-Cavallé, and E. Ahedo, "Electron cooling and finite potential drop in a magnetized plasma expansion," Phys. Plasmas 22, 053501 (2015).

${ }^{14}$ F. Cichocki, A. Domínguez-Vázquez, M. Merino, and E. Ahedo, "Hybrid 3d model for the interaction of plasma thruster plumes with nearby objects," Plasma Sources Sci. Technol. 26, 125008 (2017).

${ }^{15}$ C. Othmer, K. Glassmeier, U. Motschmann, J. Schüle, and C. Frick, "Threedimensional simulations of ion thruster beam neutralization," Phys. Plasmas $\mathbf{7}$, 5242-5251 (2000).

${ }^{16}$ A. Wheelock, D. Cooke, and N. A. Gatsonis, "Investigation of ion beam neutralization processes with 2D and 3D PIC simulations," Comput. Phys. Commun. 164, 336-343 (2004).

${ }^{17} \mathrm{~L}$. Brieda and J. Wang, "Modeling of ion thruster beam neutralization using a fully kinetic ES-PIC code," AIAA Paper No. 2005-4045, 2005.

${ }^{18} \mathrm{~J}$. Wang, O. Chang, and Y. Cao, "Electron-ion coupling in mesothermal plasma beam emission: Full particle PIC simulations," IEEE Trans. Plasma Sci. 40, 230-236 (2012).

${ }^{19}$ H. Usui, A. Hashimoto, and Y. Miyake, "Electron behavior in ion beam neutralization in electric propulsion: Full particle-in-cell simulation," J. Phys.: Conf. Ser. 454, 012017 (2013).
${ }^{20} \mathrm{Y}$. Hu and J. Wang, "Electron properties in collisionless mesothermal plasma expansion: Fully kinetic simulations," IEEE Trans. Plasma Sci. 43, 2832-2838 (2015).

${ }^{27} \mathrm{Y}$. $\mathrm{Hu}$ and J. Wang, "Fully kinetic simulations of collisionless, mesothermal plasma emission: Macroscopic plume structure and microscopic electron characteristics," Phys. Plasmas 24, 033510 (2017).

${ }^{22} \mathrm{Y}$. Hu and J. Wang, "Expansion of a collisionless hypersonic plasma plume into a vacuum," Phys. Rev. E 98, 023204 (2018).

${ }^{23}$ J. Polansky, J. Wang, and N. Ding, "Experimental investigation on plasma plume potential,” IEEE Trans. Plasma Sci. 41, 3438-3447 (2013).

${ }^{24}$ J. Wang and D. E. Hastings, "Ionospheric plasma flow over large high-voltage space platforms. I: Ion-plasma-time scale interactions of a plate at a zero-angle of attack," Phys. Fluids B 4, 1597-1614 (1992).

${ }^{25} \mathrm{~J}$. Wang and D. E. Hastings, "Ionospheric plasma flow over large high-voltage space platforms. II: The formation and structure of plasma wake," Phys. Fluids B 4, 1615-1629 (1992).

${ }^{26}$ B. W. Longmier, E. A. Bering, M. D. Carter, L. D. Cassady, W. J. Chancery, F. C. Diaz, T. W. Glover, N. Hershkowitz, A. V. Ilin, G. Mccaskill et al., "Ambipolar ion acceleration in an expanding magnetic nozzle," Plasma Sources Sci. Technol. 20, 015007 (2011).

${ }^{27}$ W. Y. L. Ling, Z. Zhang, H. Tang, X. Liu, and N. Wang, "In-plume acceleration of leading-edge ions from a pulsed plasma thruster," Plasma Sources Sci. Technol. 27, 104002 (2018). 